\title{
$\Rightarrow$ DIABETES
}

\section{Omega-6 PUFAs and T2DM}

The influence of the main dietary omega-6 polyunsaturated fatty acid (PUFA) linoleic acid and its metabolite arachidonic acid on the incidence of type 2 diabetes mellitus (T2DM) remains contentious. A new study now reports that high levels of linoleic acid, but not arachidonic acid, are associated with a lower risk of T2DM.

The investigators established the Fatty Acids and Outcomes Research Consortium (FORCE), bringing together 20 prospective cohort studies across 10 countries involving 39,740 adults that had assessed T2DM incidence and measured levels of biomarkers for omega- 6 PUFAs. "This is the largest and most detailed assessment of biomarkers of omega-6 PUFAs and the incidence of T2DM," explains lead investigator
Jason $\mathrm{Wu}$. The relative risk of T2DM was assessed prospectively for each study and pooled analyses of these data were performed across all studies and for each lipid compartment.

During a follow-up of 366,073 person-years, 4,347 participants developed T2DM. In the multivariate-adjusted pooled analyses, baseline levels of linoleic acid were inversely and significantly associated with T2DM incidence overall (risk ratio (RR) $0.65,95 \% \mathrm{Cl}$ $0.60-0.72, P<0.0001$ ) and across different lipid compartments. Conversely, baseline arachidonic acid levels were not significantly associated with the risk of T2DM across all studies (RR 0.96, $95 \% \mathrm{Cl}$ $0.88-1.05, P=0.38$ ) or in separate lipid compartments. Importantly, these associations were not significantly modified by prespecified demographic, anthropometric or biological sources of heterogeneity.

"Our novel findings suggest that linoleic acid might have long-term benefits for preventing the onset of T2DM, supporting clinical recommendations to increase dietary intake of linoleic acid-rich vegetable oils," concludes Wu. "Whether increased intake of linoleic acid leads to decreased incidence of T2DM should also be tested in randomized controlled clinical trials."

Conor A. Bradley

ORIGINAL ARTICLE Wu, J. H. Y. et al. Omega-6 fatty acid biomarkers and incident type 2 diabetes: pooled analysis of individual-level data for 39740 adults from 20 prospective cohort studies. Lancet Diabetes Endocrinol. http://dx.doi.org/10.1016/ S2213-8587(17)30307-8 (2017) 